Research Paper

\title{
Tumor Volume Reduction Rate Predicts Pathologic Tumor Response of Locally Advanced Rectal Cancer Treated with Neoadjuvant Chemotherapy alone: Results from a Prospective Trial
}

Jian Xiao ${ }^{1 \dagger}$, Zerong Cai²†, Wenyun Li ${ }^{3 \dagger}$, Zuli Yang ${ }^{2}$, Jiaying Gong, ${ }^{4}$ Yan Huang 5 , Yanhong Deng1, Xiaojian $\mathrm{Wu}^{2}$, Lei Wang2, Junsheng Peng ${ }^{2}$, Donglin Ren², Ping Lan², Jianping Wang ${ }^{2}$

1. Department of Medical Oncology, The Sixth Affiliated Hospital, Sun Yat-sen University, Guangzhou 510655, P.R. China

2. Department of Colorectal Surgery, The Sixth Affiliated Hospital, Sun Yat-sen University, Guangzhou 510655, P.R. China

3. Centre for Quantitative Medicine, Duke-NUS Graduate Medical School, National University of Singapore, 169856, Singapore

4. Department of Radiology, The Sixth Affiliated Hospital, Sun Yat-sen University, Guangzhou 510655, P.R. China

5. Department of Pathology, The Sixth Affiliated Hospital, Sun Yat-sen University, Guangzhou 510655, P.R. China

† Co-first authors: Jian Xiao, Zerong Cai and Wenyun Li contributed equally to this study.

$\triangle$ Corresponding author: Jianping Wang, M.D. Ph.D., Department of Colorectal Surgery, The Sixth Affiliated Hospital, Sun Yat-sen University, 26 Yuancun Erheng Road, Guangzhou 510655, P.R. China. Email: wangjianping_sysu@163.com

() 2015 Ivyspring International Publisher. Reproduction is permitted for personal, noncommercial use, provided that the article is in whole, unmodified, and properly cited. See http://ivyspring.com/terms for terms and conditions.

Received: 2015.01.31; Accepted: 2015.04.15; Published: 2015.05.26

\begin{abstract}
Purpose: To evaluate tumor volume reduction rate (TVRR) measured by three-dimensional region-of-interest (3D-ROI) magnetic resonance (MR) volumetry in predicting pathological tumor response of preoperative chemotherapy alone for locally advanced rectal cancer (LARC).

Methods: LARC patients who received neoadjuvant chemotherapy only from a prospective and randomized trial were recruited. Tumor volumes were measured with 3D-ROI MR volumetry. TVRR was determined using the equation TVRR $=\left(V_{\text {Pre-Therapy }}-V_{\text {Post-Therapy })} / V_{\text {Pre-Therapy }} \times 100 \%\right.$ Correlation between TVRR and clinical or pathological characteristics and predictive value of TVRR for pathological tumor response in terms of Tumor Regression Grade (TRG), T downstage, $\mathrm{N}$ downstage and overall downstage were analyzed.

Results: 80 eligible cases of LARC were included in our study with TVRR of $(51.7 \pm 25.1) \%$. TVRR was higher in well-differentiated tumors compared with poor-differentiated tumors $(P=0.040)$. TVRR was found to be related with TRG $(P<0.001)$, T downstage $(P<0.001)$ and overall downstage $(P<0.001)$. Risk of achieving TRG $2 / 3$ decreased to $57.5 \%(P=0.002)$ and odds of achieving overall downstage increase to $179.3 \%(P<0.001)$ when TVRR increased by every $10 \%$. A sensitivity of 0.704 and specificity of 0.804 were calculated when ROC curve was constructed to predict TRG using TVRR with a cutoff of $65 \%$.

Conclusion: TVRR is correlated with TRG and overall downstage significantly in LARC patients treated with preoperative chemotherapy alone and shows great value in predicting favorable TRG and overall downstage with good sensitivity and specificity. It could be considered as a promising parameter candidate for efficacy evaluation.
\end{abstract}

Key words: Rectal Cancer, Neoadjuvant Chemotherapy, Chemotherapeutic Efficacy, TVRR

\section{Introduction}

Rectal cancer is one of the most common malignancies in the world with an annual incidence of 40,000 thousands new cases in United States [1]. In
China, around 100,000 new cases of rectal cancer were diagnosed every year and locally advanced rectal cancer (LARC) accounted for half of all the rectal 
cancer patients [2].

Neoadjuvant chemoradiotherapy has been established as the standard treatment for LARC due to the evidence of improved local control and higher probability of anal sphincter preservation [3, 4]. But the short-term and long-term toxicities caused by radiotherapy and the advent of total mesorectal excision significantly diminish the benefit of neoadjuvant chemoradiotherapy. Results of several trials [5-7] indicated that neoadjuvant chemotherapy alone might be an alternative treatment option for chemoradiotherapy of LARC, and show promising pathological tumor response (PTR) including tumor regression grade and overall downstage. PTR was proved to be of important prognostic value [8] .

Tumor volume reduction rate (TVRR), which was developed with the application of three dimensional (3D) region of interest (ROI) magnetic resonance (MR) volumetry, had been investigated in predicting efficacy of neoadjuvant chemoradiotherapy for LARC [9] , and proved to be associated with PTR and overall survival. However, all these studies were retrospective analysis and all recruited patients received radiotherapy. Tumor response and shrinkage of LARC to radiotherapy or chemotherapy varied according to the extent of inflammation, edema and perirectal desmoplastic reactions [10]. Therefore, the predictive value of TVRR in LARC patients with neoadjuvant chemotherapy alone requires an independent validation.

Based on a prospective randomized trial (FOWARC, NCT 01211210) investigating the efficacy of neoadjuvant chemotherapy alone in LARC, we performed this study to prospectively explore the value of TVRR in predicting efficacy of preoperative chemotherapy alone for LARC patients through 3D ROI MR volumetry.

\section{Method and Materials}

\section{Patients}

This study was conducted basing on a prospective trial which was registered in cinicaltrial.gov (FOWARC, NCT 01211210). The study protocol was approved by the institutional review board ethics committee and signed inform consent was obtained from every patient included.

Patients with pathologically confirmed rectal adenocarcinoma within $12 \mathrm{~cm}$ from the anal verge were to be screened. The patients should be 18-75 years old with Eastern Cooperative Oncology Group (ECOG) performance status (PS) score of 0-2. Adequate hematologic, hepatic and renal functions were required. Patients with enhanced pelvic MR based cT3-T4 and/or cN+ without evidence of synchronous metastatic disease confirmed by enhanced chest-abdominal computed tomography (CT) were eligible for the study. Patients with previous or concurrent malignancy or any anti-cancer therapy were excluded. Patients who were allergic to platinum drugs or fluorouracil were also ineligible for this study.

\section{Treatment}

Participants were to receive four cycles of mFOLFOX6 (Oxaliplatin 85mg/ $\mathrm{m}^{2}$ ivdrip 2 hours, D1; Leucovorin $400 \mathrm{mg} / \mathrm{m}^{2}$ ivdrip 2 hours, D1; Fluorouracil $400 \mathrm{mg} / \mathrm{m}^{2}$ iv followed by fluorouracil $2400 \mathrm{mg} / \mathrm{m}^{2}$ civ 48 hours, D1; repeat every 2 weeks) before operation. Total mesorectal excision was the standard procedure for this study and scheduled to be performed 2-4 weeks after completion of neoadjuvant chemotherapy. Creation of a temporary diverting stomy was at the discretion of the primary surgeon [11].

\section{MR Volumetry and Pathological Evaluation}

Enhanced pelvic MR scan was performed before chemotherapy and one week before surgery respectively. All the 3D-ROI MR volumetry was performed using same protocol. The cross-sectional lesion areas were measured on axial T2-weighted images by manually tracing the lesion boundaries. Manual tracing of the lesion was performed by two radiologists. The contour of cross-sectional lesions was defined as intermediate signal intensity areas that differed from the normal adjacent rectal wall. The volumes of lesions were automatically calculated by summing each of the cross-sectional volumes (multiplying cross-sectional area by section thickness) using the Advantage Workstation, version 4.0 (General Electric Medical Systems, New York, NY). TVRR was determined using equation TVRR = $\left(V_{\text {Pre-Therapy }}-V_{\text {Post-Therapy }}\right) / V_{\text {Pre-Therapy }} \times 100 \% \quad\left(V_{\text {Pre-Therapy }}\right.$ represents for pre-chemotherapy tumor volume, $V_{\text {Post-Therapy }}$ represents for post-chemotherapy tumor volume). Positive lymph node involvement was defined as a lymph node $\geq 0.5 \mathrm{~cm}$ in the smallest diameter observed by MRI.

After surgery, the pathologic stage was determined according to the TNM staging system of the American Joint Committee on Cancer version 7 [12]. Tumor downstaging was assessed by comparing preoperative clinical stage (cT and $\mathrm{cN}$ stage) with postoperative pathologic stage (ypT and ypN stage) [13]. T downstage was defined as from cT3 to ypT2-T0, or from cT4 to ypT3-T0. N downstage was defined as from cN1-2 to ypN0. Overall downstage was defined as from any stage to ypT0-2N0. Tumor regression grade (TRG) was defined with Ryan's criteria[14] as follows: Grade 0, no viable cancer cells 
(complete regression); Grade 1, single cells or small groups of cancer cells; Grade 2, residual cancer outgrown by fibrosis; Grade 3, residual cancer outgrown by fibrosis or no fibrosis with extensive residual cancer. Regression grading involved both the primary tumor and regional lymph nodes.

\section{Statistical Analyses}

For continuous variables, the data were expressed as mean \pm SD or median (Minimum-Maximum). Statistical significance of the association between TVRR and characteristic was estimated using $t$-test or chi-square test. Logistic regression analysis was used to analyze the correlation between the TVRR and pathological response indexes. Receiver operating characteristics (ROC) curves were constructed to obtain area under the curve (AUC) and to calculate sensitivity, specificity, positive predictive value (PPV), and negative predictive value (NPV) at specific cutoff points (maximizing sensitivity, specificity or both). All the analysis was performed using SPSS version17.0 or SAS version 9.1. Two sided $P<$ 0.05 was considered statistically significant.

\section{Results}

\section{Patient Characteristics}

From October 2010 to September 2013, a total of eighty-seven cases of LARC patients met the inclusion criteria. Seven patients were excluded due to withdrawal of informed consent $(n=3)$, protocol deviation $(n=3)$, or received surgery elsewhere $(n=1)$. Eighty patients completed the entire neoadjuvant chemotherapy and radical resection of tumor. As shown in Table 1, patients had a median age of 53 (25-73) years. Fifty-five $(68.8 \%)$ were males and the median distance from anal verge was $6.2(2.0-12.0) \mathrm{cm}$. Seventy-four $(92.5 \%)$ patients were well/moderately differentiated.
Sixty-two (77.5\%) patients shared a normal pre-treatment serum CEA level.

According to the MR scanning results, cT2 was seen in $1(1.3 \%)$ case, cT3 were seen in $73(91.3 \%)$ cases and $6(7.5 \%)$ cases had cT4. Thirty $(37.5 \%)$ cases were cN1, $23(28.8 \%)$ cases were cN2 and $27(33.8 \%)$ cases were $\mathrm{cN} 0$ according to the assessment of regional lymph node. Finally, all the patients underwent radical tumor resection with 59 cases (73.8\%) of Dixon's procedure, 7 cases $(8.8 \%)$ of Park's procedure and 14 cases $(17.4 \%)$ of Mile's procedure.

\section{Pathological Results and MR Volumetry}

Table 2 showed the pathological results of the 80 patients after operation. According to the AJCC TNM staging system, $5(6.3 \%)$ cases were to have ypStage 0 , $20(25.0 \%)$ cases ypStage I, $36(45.0 \%)$ cases ypStage II and 19 cases $(23.7 \%)$ ypStage III. 3 cases $(3.7 \%)$ of lymphovascular invasion, 9 cases $(11.3 \%)$ of perineural invasion and 9 cases $(11.3 \%)$ of extra nodal tumor deposits were found. 1 case $(1.2 \%)$ found a positive circumferential resection margin. MR volumetry was illustrated as Figure 1. The mean pre-chemotherapy tumor volume was $35.0 \mathrm{~cm}^{3} \pm 38.7 \mathrm{~cm}^{3}$, and the mean post-chemotherapy tumor volume was $16.5 \mathrm{~cm}^{3} \pm 16.2 \mathrm{~cm}^{3}$. Mean TVRR of $51.7 \%( \pm 25.1 \%)$ was calculated according to the equation, and 3 cases of patients came out with a negative TVRR since their tumor volumes were enlarged after chemotherapy according to volumetry. Pathological TRG assessment showed 5 cases $(6.3 \%)$ in TRG0, 20 cases $(23.8 \%)$ in TRG1, 30 cases (37.5\%) in TRG2 and 25 cases (31.2\%) in TRG 3. Compared with the pre-chemotherapy stage, 24 cases $(30.0 \%)$ achieved $\mathrm{T}$ downstage, 43 cases $(53.8 \%)$ achieved $\mathrm{N}$ downstage and 25 cases $(31.2 \%)$ achieved overall downstage.

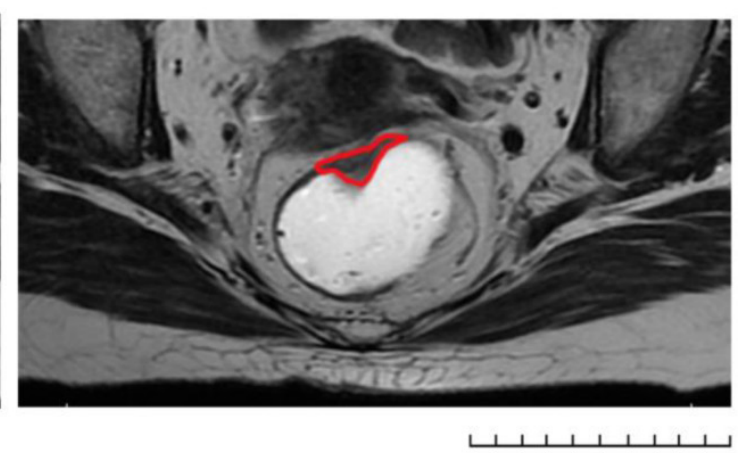

Post-Chemotherapy

Figure 1. Represent MRI graphs of rectal cancer before and after chemotherapy in the same transverse section: Region of Interest was sketched in red line on T2-weighted images. 
Table 1. Characteristics of Patients $(n=80)$

\begin{tabular}{ll}
\hline Characteristics & Number $(\%) /$ Average \pm SD \\
\hline Gender & $55(68.8 \%)$ \\
Male & $25(31.2 \%)$ \\
Female & $53(25 \sim 73)$ \\
Age & $6.2(2.0 \sim 12.0)$ \\
Tumor Distance From Anal Verge & $31(38.8 \%)$ \\
$\leq 5 \mathrm{~cm}$ & $49(61.2 \%)$ \\
$>5 \mathrm{~cm}$ & \\
Differentiation & $74(92.5 \%)$ \\
Well/Moderate & $6(7.5 \%)$ \\
Poor/mucinous adenocarcinoma & \\
Serum CEA & $62(77.5 \%)$ \\
$\leq 5$ ng/ml & $18(22.5 \%)$ \\
$>5$ ng/ml & \\
cT Stage & $1(1.3 \%)$ \\
cT2 & $73(91.2 \%)$ \\
cT3 & $6(7.5 \%)$ \\
cT4 & \\
cN Stage & $27(33.8 \%)$ \\
cN- & $53(66.2 \%)$ \\
cN+ & \\
Procedure & $59(73.8 \%)$ \\
Dixon's & $7(8.8 \%)$ \\
Park's & $14(17.4 \%)$ \\
Mile's & \\
\hline & \\
\hline
\end{tabular}

Table 2. Pathological and Volumetry Result of Patients

\begin{tabular}{|c|c|}
\hline Pathological/Volumetry Index & Number (\%) / Average \pm SD \\
\hline \multicolumn{2}{|l|}{ pT Stage } \\
\hline урт0 & $5(6.2 \%)$ \\
\hline урT1-2 & $23(28.8 \%)$ \\
\hline урТ3 & $51(63.8 \%)$ \\
\hline урT4 & $1(1.2 \%)$ \\
\hline \multicolumn{2}{|l|}{ pN Stage } \\
\hline ypNo & $61(76.3 \%)$ \\
\hline ypN1 & $9(11.3 \%)$ \\
\hline ypN2 & $10(12.4 \%)$ \\
\hline \multicolumn{2}{|l|}{ pTNM Stage } \\
\hline $\mathrm{pCR}$ & $5(6.3 \%)$ \\
\hline Stage I & $20(25.0 \%)$ \\
\hline Stage II & $36(45.0 \%)$ \\
\hline Stage III & $19(23.7 \%)$ \\
\hline \multicolumn{2}{|l|}{ Lymphovascular Invasion } \\
\hline Yes & $3(3.7 \%)$ \\
\hline No & $77(96.3 \%)$ \\
\hline \multicolumn{2}{|l|}{ Perineural Invasion } \\
\hline Yes & $9(11.3 \%)$ \\
\hline No & $71(88.7 \%)$ \\
\hline \multicolumn{2}{|l|}{ Extra Nodal Tumor Deposits } \\
\hline Yes & $9(11.3 \%)$ \\
\hline No & $71(88.7 \%)$ \\
\hline \multicolumn{2}{|l|}{ CRM } \\
\hline$\leq 1 \mathrm{~mm}$ & $1(1.2 \%)$ \\
\hline$>1 \mathrm{~mm}$ & $71(98.8 \%)$ \\
\hline$V_{\text {Pre-Therapy }}\left(\mathrm{cm}^{3}\right)$ & $35.0 \pm 38.7$ \\
\hline$V_{\text {Post-Therapy }}\left(\mathrm{cm}^{3}\right)$ & $16.5 \pm 16.2$ \\
\hline TVRR (\%) & $51.7 \pm 25.1$ \\
\hline \multicolumn{2}{|l|}{ T Downstage } \\
\hline Yes & $24(30.0 \%)$ \\
\hline No & $56(70.0 \%)$ \\
\hline \multicolumn{2}{|l|}{ N Downstage } \\
\hline Yes & $43(53.8 \%)$ \\
\hline No & $37(46.2 \%)$ \\
\hline \multicolumn{2}{|l|}{ Overall Downstage } \\
\hline Yes & $25(31.2 \%)$ \\
\hline No & $55(68.8 \%)$ \\
\hline \multicolumn{2}{|l|}{ TRG } \\
\hline 0 & $5(6.3 \%)$ \\
\hline 1 & $20(25.0 \%)$ \\
\hline 2 & $30(37.5 \%)$ \\
\hline 3 & $25(31.2 \%)$ \\
\hline
\end{tabular}

Table 3. TVRR according to Patient Characteristics and Pathological Results

\begin{tabular}{|c|c|c|c|}
\hline Characteristics/Index & Number $(\%)$ & TVRR & $P$ Value \\
\hline Gender & & & 0.283 \\
\hline Male & $55(68.8 \%)$ & $47.2 \pm 22.7$ & \\
\hline Female & $25(31.2 \%)$ & $53.7 \pm 26.1$ & \\
\hline Age & & & 0.318 \\
\hline$\leq 60$ & $50(62.5 \%)$ & $53.9 \pm 23.3$ & \\
\hline$>60$ & $30(37.5 \%)$ & $48.0 \pm 27.9$ & \\
\hline Tumor Distance From Anal Verge & & & 0.445 \\
\hline$\leq 5 \mathrm{~cm}$ & $31(38.8 \%)$ & $54.4 \pm 25.3$ & \\
\hline$>5 \mathrm{~cm}$ & $49(61.2 \%)$ & $50.0 \pm 25.1$ & \\
\hline Differentiation & & & 0.040 \\
\hline Well/Moderate & $74(92.5 \%)$ & $53.3 \pm 23.9$ & \\
\hline Poor/mucinous adenocarcinoma & $6(7.5 \%)$ & $31.5 \pm 33.3$ & \\
\hline Serum CEA & & & 0.394 \\
\hline$\leq 5 \mathrm{ng} / \mathrm{ml}$ & $62(77.5 \%)$ & $53.0 \pm 24.4$ & \\
\hline$>5 \mathrm{ng} / \mathrm{ml}$ & $18(22.5 \%)$ & $47.2 \pm 27.8$ & \\
\hline cT Stage & & & 0.617 \\
\hline cT2-3 & $74(92.5 \%)$ & $51.3 \pm 25.6$ & \\
\hline cT4 & $6(7.5 \%)$ & $56.6 \pm 14.7$ & \\
\hline cN Stage & & & 0.859 \\
\hline $\mathrm{cN}-$ & $27(33.8 \%)$ & $52.4 \pm 25.7$ & \\
\hline $\mathrm{cN}+$ & $53(66.2 \%)$ & $51.3 \pm 25.0$ & \\
\hline cTNM Stage & & & 0.859 \\
\hline II & $27(33.8 \%)$ & $52.4 \pm 25.7$ & \\
\hline III & $53(66.2 \%)$ & $51.3 \pm 25.0$ & \\
\hline pT Stage & & & 0.001 \\
\hline pT0-2 & $28(35.0 \%)$ & $64.7 \pm 21.0$ & \\
\hline pT3-4 & $52(65.0 \%)$ & $45.2 \pm 24.9$ & \\
\hline pN Stage & & & 0.063 \\
\hline $\mathrm{pN}-$ & $61(76.3 \%)$ & $54.6 \pm 23.3$ & \\
\hline $\mathrm{pN}+$ & $19(23.7 \%)$ & $42.3 \pm 28.9$ & \\
\hline pTNM Stage & & & $<0.001$ \\
\hline pCR/I Stage & $25(31.3 \%)$ & $66.3 \pm 16.5$ & \\
\hline II/ III Stage & $55(68.7 \%)$ & $45.0 \pm 25.7$ & \\
\hline Lymphovascular Invasion & & & 0.056 \\
\hline Yes & $3(3.8 \%)$ & $24.6 \pm 34.4$ & \\
\hline No & $77(96.2 \%)$ & $52.7 \pm 24.4$ & \\
\hline Perineural Invasion & & & 0.564 \\
\hline Yes & $9(11.3 \%)$ & $47.0 \pm 10.6$ & \\
\hline No & $71(88.7 \%)$ & $52.3 \pm 26.4$ & \\
\hline Extra Nodal Tumor Deposits & & & 0.416 \\
\hline Yes & $9(11.3 \%)$ & $45.2 \pm 27.9$ & \\
\hline No & $71(88.7 \%)$ & $52.5 \pm 24.9$ & \\
\hline CRM & & & NA \\
\hline$\leq 1 \mathrm{~mm}$ & $1(1.3 \%)$ & -31.7 & \\
\hline$>1 \mathrm{~mm}$ & $79(98.7 \%)$ & $52.7 \pm 23.4$ & \\
\hline
\end{tabular}

\section{Relationship of TVRR and Patient's Clinical and Pathological Characteristics}

We analyzed the difference of TVRR between patients' clinical variables. As showed in the Table 3, we found no significant difference of TVRR between gender, age, tumor location, cT stage, cTNM stage and serum CEA level. However, the TVRR of rectal patients with well/moderate differentiation adenocarcinoma was significantly higher than the patient's in poor differentiation group ( $51.3 \%$ vs $31.5 \%, P=0.040$ ). Furthermore, TVRR showed statistical different in different $\mathrm{T}$ stages $(P<0.05)$ and the post analysis showed the difference between pT1 and pT3, pT2 and T3 possessed significant difference (pT1 vs pT3: $70.04 \%$ vs $45.22 \%, P=0.012$; $\mathrm{pT} 2$ vs $\mathrm{pT} 3,64.10 \%$ vs $45.22 \%, P=0.007)$. The TVRR of patients with stage I was higher than those with stage II $(69.47 \%$ vs 
$46.45 \%, P<0.001)$ and stage III $(69.47 \%$ vs $42.35 \%$, $P<0.001)$. On the other hand, the TVRR showed no significance according to lymphovascular invasion, perineural invasion and extra nodal tumor deposits. The patient with positive circumferential resection margin possessed a TVRR of $-31.67 \%$, which indicated the enlargement of tumor volume after chemotherapy.

\section{Relationship of TVRR and Patient's Thera- peutic Response}

We analyzed the difference of TVRR, $\mathrm{V}_{\text {Pre-Therapy, }}$ and $V_{\text {Post-Therapy }}$ according to the patients' TRG, $T$ downstage, $\mathrm{N}$ downstage and overall downstage (Table 4). Tumor volume before chemotherapy of the patients who achieved overall downstage was smaller than the patients' who did not $\left(19.54 \mathrm{~cm}^{3} \mathrm{vs} 42.00 \mathrm{~cm}^{3}\right.$, $P=0.015)$, and so was the volume after chemotherapy $(6.71 \mathrm{~cm} 3$ vs $20.98 \mathrm{~cm} 3, P<0.001)$. However, the tumor volume before/after chemotherapy did not differ when they were divided by TRG, T downstage and $\mathrm{N}$ downstage. TVRR of the patients who achieved TRG $0 / 1$ was higher than patients' who were TRG 2/3 (65.91\% vs $46.20 \%, P<0.001)$. TVRR of the patients who achieved $\mathrm{T}$ downstage was higher than patients' who did not $(66.28 \%$ vs $48.18 \%, P<0.001)$. And similar tendency was observed when TVRR was compared according to overall downstage $(66.03 \%$ vs $16.50 \%$, $P<0.001)$.

\section{Clinical Value of TVRR in Predicting Tumor Therapeutic Response}

Using logistic regression analysis, we tried to assess the value of tumor volume and TVRR in predicting therapeutic response of neoadjuvant chemotherapy in rectal cancer. As the result showed in Table 5 , the tumor volumes before/after chemotherapy were both predictors of overall downstage in rectal cancer. The odds of patient achieving overall downstage decreased into 0.655 time (95\% CI: 0.477 0.901, $P=0.009)$ when $V_{\text {Pre-Therapy }}$ increased by $10 \mathrm{~cm}^{3}$, and this odds decreased into 0.266 (95\% CI: 0.110 0.646, $P=0.003)$ time when $V_{\text {Post-Therapy }}$ increased by $10 \mathrm{~cm}^{3}$. Moreover, the logistic regression analysis suggested that higher TVRR predict favor TRG and overall downstage in rectal cancer. When the TVRR increased by every $10 \%$, risk of achieving TRG $2 / 3$ decreased into 0.575 (95\% CI: 0.407 0.810, $P=0.002)$ time and odds of achieving overall downstage increase into 1.793 (95\% CI: 1.258 2.555, $P<0.001$ ) times.

ROC curve was constructed to evaluate the value of TVRR in predicting TRG and overall downstage. An optimal cutoff of $65 \%$ was calculated to maximize both sensitivity and specificity of TVRR. And using this cutoff, TVRR came out with good sensitivity and specialty in predicting TRG and overall downstage when dichotomizing patients, as showed in Table 6.

Table 4. Tumor Volumetry Results according to Pathological Response Index

\begin{tabular}{|c|c|c|c|c|c|c|c|c|}
\hline \multirow[t]{2}{*}{ Volumetry } & \multicolumn{2}{|c|}{ TRG } & \multicolumn{2}{|c|}{ T Downstage } & \multicolumn{2}{|c|}{ N Downstage } & \multicolumn{2}{|c|}{ Overall Downstage } \\
\hline & $0 / 1$ & $2 / 3$ & Yes & No & Yes & No & Yes & No \\
\hline $\mathrm{V}_{\text {Pre-Therapy }}$ & $40.58 \pm 57.05$ & $32.43 \pm 26.85$ & $36.44 \pm 57.58$ & $34.35 \pm 27.58$ & $37.79 \pm 46.29$ & $31.70 \pm 27.60$ & $19.54 \pm 14.96$ & $42.00 \pm 43.91$ \\
\hline$P$ Value & 0.386 & & 0.827 & & 0.486 & & 0.015 & \\
\hline$V_{\text {Post-Therapy }}$ & $13.43 \pm 15.04$ & $17.93 \pm 16.59$ & $13.26 \pm 16.66$ & $17.92 \pm 15.90$ & $15.38 \pm 13.82$ & $17.85 \pm 18.64$ & $6.71 \pm 6.68$ & $20.98 \pm 17.26$ \\
\hline$P$ Value & 0.251 & & 0.240 & & 0.498 & & $<0.001$ & \\
\hline TVRR & $65.91 \pm 17.68$ & $46.20 \pm 25.44$ & $59.98 \pm 20.75$ & $48.11 \pm 26.13$ & $54.75 \pm 20.59$ & $48.09 \pm 29.41$ & $66.28 \pm 45.03$ & $16.50 \pm 25.65$ \\
\hline$P$ Value & $<0.001$ & & 0.052 & & 0.240 & & $<0.001$ & \\
\hline
\end{tabular}

Table 5. Logistic Regression of TVRR in Predicting Pathological Response

\begin{tabular}{|c|c|c|c|c|c|c|c|c|}
\hline \multirow[t]{2}{*}{ Volumetry* } & \multicolumn{2}{|c|}{ TRG (0/1 vs $2 / 3)$} & \multicolumn{2}{|c|}{ T Downstage } & \multicolumn{2}{|c|}{ N Downstage } & \multicolumn{2}{|c|}{ Downstage Stage } \\
\hline & OR $(95 \% \mathrm{CI})$ & $P$ & OR $(95 \% \mathrm{CI})$ & $P$ & OR $(95 \%$ CI $)$ & $P$ 值 & OR $(95 \% \mathrm{CI})$ & $P$ \\
\hline$V_{\text {Pre-Therapy }}$ & $0.950(0.843 \sim 1.070)$ & 0.399 & $1.014(0.899 \sim 1.143)$ & 0.825 & $1.046(0.921 \sim 1.188)$ & 0.491 & $0.655(0.477 \sim 0.901)$ & 0.009 \\
\hline$V_{\text {Post-Therapy }}$ & $1.214(0.871 \sim 1.690)$ & 0.252 & $0.817(0.582 \sim 1.146)$ & 0.242 & $0.990(0.690 \sim 1.196)$ & 0.494 & $0.266(0.110 \sim 0.646)$ & 0.003 \\
\hline TVRR & $0.575(0.407 \sim 0.810)$ & 0.002 & $1.263(0.991 \sim 1.611)$ & 0.060 & 1.015 (0.930 1.336) & 0.240 & 1.793 (1.258 2.555) & 0.001 \\
\hline
\end{tabular}

*Volume change by every $10 \mathrm{~cm}^{3}$ and TVRR changes by every $10 \%$.

Table 6. Sensitivity and Specificity Parameters of TVRR in predicting Pathological Response

\begin{tabular}{|c|c|c|c|c|c|}
\hline Pathological Response Index & AUC & Sensitivity & Specificity & Positive Predictive Value & Negative Predictive Value \\
\hline TRG (0/1 vs $2 / 3)$ & 0.761 & 0.704 & 0.804 & 0.792 & 0.719 \\
\hline T Downstage & 0.662 & 0.617 & 0.756 & 0.771 & 0.596 \\
\hline N Downstage & 0.540 & 0.508 & 0.625 & 0.688 & 0.439 \\
\hline Overall Downstage & 0.779 & 0.646 & 0.702 & 0.646 & 0.702 \\
\hline
\end{tabular}




\section{Discussion}

In this article, we exposed the clinical value of TVRR in predicting therapeutic response of preoperative chemotherapy alone in LARC, basing on a prospective and randomized trial. Higher TVRR was associated with better differentiation of tumor and predicted favorable TRG, T downstage and overall downstage in rectal cancer. TVRR act as a predictor of chemotherapeutic efficacy after neoadjuvant chemotherapy alone with good sensitivity and specialty in rectal cancer.

Multidisciplinary treatment has been considered as the standard therapy for LARC. The combination of surgery, preoperative chemoradiotherapy and postoperative chemotherapy improves the local control and long term survival of LARC significantly. It has been proved [15] that the treatment response of solid tumor to chemoradiotherapy correlates with the prognosis closely. Thus, the prediction of tumor response plays an important role in the comprehensive treatment plan. Recently, TVRR has been demonstrated as an early predictor of local control for head-and-neck cancer and might indicate early intervention for patients at high risk of treatment failure [16-19]. Yeo et al $[9,20]$ found TVRR associated with TRG and downstage significantly in LARC patients treated with chemoradiotherapy in retrospective studies. However, prospective clinical study is rarely seen and tumor volume may be interfered owing to the radiation-related fibrosis and edema when radiotherapy is involved. Thus, based on data of patients treated with standard chemotherapy without radiotherapy in prospective clinical trial, the interference of irradiation could be avoid and more accurate volumetry may be achieved. In patients with TVRR higher than $80 \%$ in our study, the percentage of pathological downstage or TRG 0/1 was greater than that in Yeo's study (overall downstage: $63.5 \%$ vs $50.5 \%$, TRG 0/1: $87.5 \%$ vs $39.3 \%$ ), which might be due to the inaccurate volumetry caused by irradiation and indicated that TVRR would be more precise when radiotherapy is absent.

TRG is a golden standard in assessing chemotherapy response and relates with treatment sensitivity, invasiveness and metastasis of rectal cancer [21, 22]. In this study, we proved TVRR to associate with TRG and predict pathological response in term of TRG and pathological downstage in rectal cancer patients receiving neoadjuvant chemotherapy, which is consistent with previous studies which aim at patients receiving neoadjuvant chemoradiotherapy $[9,20]$. Compared with TRG, TVRR is more time-efficient and does not rely on the specimen pathological results. In several recent clinical trials[23, 24], some surgeons selected patients with good radiotherapy response to underwent local tumor excision and came out with local control and survival rates comparable to those achieved with mesorectal excision. Moreover, it has been proposed that surgical resection might not lead to improved outcome in stage 0 rectal cancer following by neoadjuvant therapy and a wait-and-see policy was feasible in strict selection cases $[25,26]$. These concepts mean that resection procedure may be changed or even avoid if we distinguish patients with extremely good response. Therefore, TVRR could be an effective therapeutic response predictor and provide valuable information that guides appropriate surgical treatment.

In our results, TVRR did not relate to $\mathrm{N}$ downstage of rectal cancer after chemotherapy. This might contribute to the limitation of MRI scan in lymph node staging. The MR lymph node staging criteria of rectal cancer bases on size, shape and signal intensity but more than half of the metastatic nodes are less than $5 \mathrm{~mm}$ in size. New method to detect $\mathrm{N}$ stage is needed to assess $\mathrm{N}$ downstage accurately. In addition, long term data was still unavailable and the prognostic value of TVRR was yet to be investigated in the coming years.

\section{Conclusion}

Above all, in our prospective and randomized clinical trial, we used 3D-ROI MR volumetry and found significant associations between TRG, downstage and TVRR in LARC patients receiving chemotherapy but without radiotherapy. TVRR was proved to predict favor TRG and overall downstage in rectal cancer with good sensitivity and specialty.

\section{Acknowledgements}

This study was sponsored by Guangdong Province Medical Research Funding (A2014266), Guangdong Department Science \& Technology Translational Medicine Center Grant (2011A080300002) and supported by National Key Clinical Discipline.

\section{Conflict of Interest}

All the authors declare that they have no conflict of interest.

\section{References}

1. Siegel R, Naishadham D, Jemal A. Cancer Statistics, 2013. CA Cancer J Clin. 2013; 1: 11-30.

2. Chen W, Zheng R, Zhang S et al. Annual Report On Status of Cancer in China, 2010. Chin J Cancer Res. 2014; 1: 48-58.

3. Benson AR, Bekaii-Saab T, Chan E et al. Rectal Cancer. J Natl Compr Canc Netw. 2012; 12: 1528-64.

4. Kapiteijn E, Marijnen CA, Nagtegaal ID et al. Preoperative Radiotherapy Combined with Total Mesorectal Excision for Resectable Rectal Cancer. N Engl J Med. 2001; 9: 638-46.

5. Fernandez-Martos C, Pericay C, Aparicio J et al. Phase II, Randomized Study of Concomitant Chemoradiotherapy Followed by Surgery and Adjuvant Capecitabine Plus Oxaliplatin (CAPOX) Compared with Induction CAPOX Followed by Concomitant Chemoradiotherapy and Surgery in Magnetic 
Resonance Imaging-Defined, Locally Advanced Rectal Cancer: Grupo Cancer De Recto 3 Study. J Clin Oncol. 2010; 5: 859-65.

6. Allegra CJ, Yothers G, O'Connell MJ et al. Phase III Trial Assessing Bevacizumab in Stages II and III Carcinoma of the Colon: Results of NSABP Protocol C-08. J Clin Oncol. 2011; 1: 11-6.

7. Schrag D, Weiser MR, Goodman KA et al. Neoadjuvant Chemotherapy without Routine Use of Radiation Therapy for Patients with Locally Advanced Rectal Cancer: A Pilot Trial. J Clin Oncol. 2014; 6: 513-8.

8. Fokas E, Liersch T, Fietkau R et al. Tumor Regression Grading After Preoperative Chemoradiotherapy for Locally Advanced Rectal Carcinoma Revisited: Updated Results of the CAO/ARO/AIO-94 Trial. I Clin Oncol. 2014; 15: 1554-62.

9. Yeo SG, Kim DY, Park JW et al. Tumor Volume Reduction Rate After Preoperative Chemoradiotherapy as a Prognostic Factor in Locally Advanced Rectal Cancer. Int J Radiat Oncol Biol Phys. 2012; 2: e193-9.

10. Kuo LJ, Chern MC, Tsou MH et al. Interpretation of Magnetic Resonance Imaging for Locally Advanced Rectal Carcinoma After Preoperative Chemoradiation Therapy. Dis Colon Rectum. 2005; 1: 23-8.

11. Wang T, Wang J, Deng Y, Wu X, Wang L. Neoadjuvant Therapy Followed by Local Excision and Two-Stage Total Mesorectal Excision: A New Strategy for Sphincter Preservation in Locally Advanced Ultra-Low Rectal Cancer. Gastroenterol Rep (Oxf). 2014; 1: 37-43.

12. Sobin LH, Compton CC. TNM Seventh Edition: What's New, What's Changed: Communication From the International Union Against Cancer and the American Joint Committee On Cancer. Cancer. 2010; 22: 5336-9.

13. Kim NK, Baik SH, Min BS et al. A Comparative Study of Volumetric Analysis, Histopathologic Downstaging, and Tumor Regression Grade in Evaluating Tumor Response in Locally Advanced Rectal Cancer Following Preoperative Chemoradiation. Int J Radiat Oncol Biol Phys. 2007; 1: 204-10.

14. Ryan R, Gibbons D, Hyland JM et al. Pathological Response Following Long-Course Neoadjuvant Chemoradiotherapy for Locally Advanced Rectal Cancer. Histopathology. 2005; 2: 141-6.

15. Therasse P. Measuring the Clinical Response. What Does It Mean? Eur J Cancer. 2002; 14: 1817-23.

16. Mayr NA, Taoka T, Yuh WT et al. Method and Timing of Tumor Volume Measurement for Outcome Prediction in Cervical Cancer Using Magnetic Resonance Imaging. Int J Radiat Oncol Biol Phys. 2002; 1: 14-22.

17. Wang JZ, Mayr NA, Zhang D et al. Sequential Magnetic Resonance Imaging of Cervical Cancer: The Predictive Value of Absolute Tumor Volume and Regression Ratio Measured Before, During, and After Radiation Therapy. Cancer. 2010; 21: 5093-101.

18. Mayr NA, Yuh WT, Taoka T et al. Serial Therapy-Induced Changes in Tumor Shape in Cervical Cancer and their Impact On Assessing Tumor Volume and Treatment Response. AJR Am J Roentgenol. 2006; 1: 65-72.

19. Yang SN, Liao CY, Chen SW et al. Clinical Implications of the Tumor Volume Reduction Rate in Head-And-Neck Cancer During Definitive Intensity-Modulated Radiotherapy for Organ Preservation. Int J Radiat Oncol Biol Phys. 2011; 4: 1096-103.

20. Yeo SG, Kim DY, Kim TH et al. Tumor Volume Reduction Rate Measured by Magnetic Resonance Volumetry Correlated with Pathologic Tumor Response of Preoperative Chemoradiotherapy for Rectal Cancer. Int I Radiat Oncol Biol Phys. 2010; 1: 164-71.

21. Fokas E, Liersch T, Fietkau R et al. Tumor Regression Grading After Preoperative Chemoradiotherapy for Locally Advanced Rectal Carcinoma Revisited: Updated Results of the CAO/ARO/AIO-94 Trial. J Clin Oncol. 2014; 15: $1554-62$

22. Buyse M, Thirion P, Carlson RW, Burzykowski T, Molenberghs G, Piedbois P. Relation Between Tumour Response to First-Line Chemotherapy and Survival in Advanced Colorectal Cancer: A Meta-Analysis. Meta-Analysis Group in Cancer. Lancet. 2000; 9227: 373-8.

23. Bonnen M, Crane C, Vauthey JN et al. Long-Term Results Using Local Excision After Preoperative Chemoradiation Among Selected T3 Rectal Cancer Patients. Int J Radiat Oncol Biol Phys. 2004; 4: 1098-105.

24. Guerrieri M, Baldarelli M, Organetti L et al. Transanal Endoscopic Microsurgery for the Treatment of Selected Patients with Distal Rectal Cancer: 15 Years Experience. Surg Endosc. 2008; 9: 2030-5.

25. Maas M, Beets-Tan RG, Lambregts DM et al. Wait-And-See Policy for Clinical Complete Responders After Chemoradiation for Rectal Cancer. J Clin Oncol. 2011; 35: 4633-40

26. Habr-Gama A, Perez RO, Nadalin $\mathrm{W}$ et al. Operative Versus Nonoperative Treatment for Stage 0 Distal Rectal Cancer Following Chemoradiation Therapy: Long-Term Results. Ann Surg. 2004; 4: 711-7, 717-8. 